\title{
Roaming at Constant Kinetic Energy: Chesnavich's Model and the Hamiltonian Isokinetic Thermostat
}

\author{
Vladimír Krajňákł* \\ School of Mathematics \\ University of Bristol \\ Bristol BS8 $1 T W$ \\ United Kingdom \\ Gregory S. Ezra ${ }^{\dagger}$ \\ Department of Chemistry and Chemical Biology \\ Baker Laboratory \\ Cornell University \\ Ithaca, NY 14853 \\ $U S A$ \\ Stephen Wiggins \\ School of Mathematics \\ University of Bristol \\ Bristol BS8 $1 T W$ \\ United Kingdom
}

\begin{abstract}
We consider the roaming mechanism for chemical reactions under the nonholonomic constraint of constant kinetic energy. Our study is carried out in the context of the Hamiltonian isokinetic thermostat applied to Chesnavich's model for an ion-molecule reaction. Through an analysis of phase space structures we show that imposing the nonholonomic constraint does not prevent the system from exhibiting roaming dynamics, and that the origin of the roaming mechanism turns out to be analogous to that found in the previously studied Hamiltonian case.
\end{abstract}




\section{INTRODUCTION}

The roaming mechanism for chemical reactions was introduced in reference to the description of the photodissociation of formaldehyde $\mathrm{e}^{2|25| 30}$. After excitation of the formaldehyde molecule by a laser pulse, a single $\mathrm{CH}$ bond begins to stretch. Rather than proceed directly to dissociation, the hydrogen atom rotates around the molecular fragment in a region of the potential energy surface where it is subject to weak forces. The corresponding motion of the hydrogen atom was termed 'roaming'. At a later stage the roaming hydrogen atom encounters the bound hydrogen atom and undergoes an abstraction reaction. The resulting $\mathrm{H}_{2}$ molecule then separates from the $\mathrm{CO}$ fragment. This reaction is then said to occur by the roaming mechanism.

Since the pioneering formaldehyde studies a similar roaming mechanism has been observed to occur in a number of chemical reactions. Many of these reactions are described in several review articles $3 \frac{3}{5 / 19 / 24}$.

Essentially all of these roaming reactions have been studied at constant total energy. The goal of this article is to study analogous roaming reaction mechanisms at constant kinetic energy. Specifically, we study Chesnavich's model for an ion-molecule reaction subjected to a Hamiltonian isokinetic thermostat.

Chesnavich's empirical model for the ion-molecule reaction $\mathrm{CH}_{4}^{+} \rightleftharpoons \mathrm{CH}_{3}^{+}+\mathrm{H}$ was introduced in reference 6. A detailed derivation of this model can be found in reference 10 . Chesnavich's model captures many of the essential aspects of the roaming mechanism. It describes the situation where a hydrogen atom separates from the rigid $\mathrm{CH}_{3}^{+}$core and, instead of dissociating, roams in a region of nearly constant potential only to return to the core. While Chesnavich's model does not accurately describe the intramolecular abstraction and subsequent dissociation, it has nevertheless provided significant insight into the roaming process, see, for example, refernces 12,13,16, 17.

In the present work we apply a Hamiltonian version of the isokinetic thermostat to Chesnavich's model. This thermostat is not as widely used as the Nosé-Hoover thermostat, and its many variants, but the non-Hamiltonian version of the isokinetic thermostat has been developed and applied to a variety of problems of interest in chemistry by Minary et al.20121. In this thermostat, the particle momenta are subject to a nonholonomic constraint that keeps the kinetic energy constant. The resulting dynamics (if ergodic) generates a canonical 
distribution in configuration space, where the associated temperature is related to the value of the conserved kinetic energy ${ }^{23}$. A Hamiltonian version of the isokinetic thermostat was given by Dettmann ${ }^{\sqrt[823]{23}}$ (see also ${ }^{14[22}$ ).

The Hamiltonian isokinetic thermostat dynamics explored in our paper is relatively unfamiliar, although, as noted, there has been some relevant earlier work in the chemical literature ${ }^{20 \mid 21}$. The basic theory ${ }^{\sqrt[8 \mid 23]{23}}$ shows that Hamiltonian dynamics in terms of a set of variables related to the physical variables by a noncanonical tranformation, together with the use of the exponentiated potential results in a canonical distribution in the physical configuration variables (assuming, as usual, ergodic dynamics). The unfamiliar aspect of the dynamics lies in the imposition of a nonholonomic constraint on the original variables; namely, constant kinetic energy. Again, the general theory shows that the effective temperature is proprtional to the value of the conserved kinetic energy.

It is in this sense that the nonholonomic dynamics explored here for the Chesnavich model is effectively at constant temperature. We do not claim that this dynamics is the same as obtained using a Boltzmann distribution for the physical momenta; nevertheless, one point of our paper is that the phase space structure of the nonholonomic system can in this case be analyzed using methods employed for the microcanonical case, and an analogous roaming mechanism explored.

For our purposes the Hamiltonian formulation of the isokinetic thermostat then has several advantages. There has been considerable development in our understanding of phase space structures governing chemical reaction dynamics in recent years, see, for example, references 26-29. This phase space structure approach has been applied to the Hamiltonian isokinetic thermostat formalism $\frac{79}{7}$. In this paper we continue these developments in our study of the Hamiltonian phase space structure associated with the roaming mechanism as exhibited in Chesnavich's model subjected to a Hamiltonian isokinetic thermostat.

This paper is outlined as follows: In Sec. II we discuss the Hamiltonian formulation of the isokinetic thermostat for a general system of the form 'kinetic + potential'. In Sec. III we introduce Chesnavich's model for roaming in $\mathrm{CH}_{4}^{+}$, and discuss the dynamical origin of roaming in terms of families of unstable periodic orbits and their associated invariant manifolds. In Sec. IV] we introduce the Hamiltonian for Chesnavich's model subject to an isokinetic thermostat. In the absence of self-retracing orbits for the thermostatted system, we find two families of periodic orbits that are relevant to the roaming phenomenon. Analysis 
of escape times and Lagrangian descriptors shows that roaming does indeed occur in the thermostatted system, via a mechanism analogous to that found in the Hamiltonian case. Sec. V] concludes.

\section{THE HAMILTONIAN ISOKINETIC THERMOSTAT}

\section{A. Thermostatted Hamiltonian}

We consider a Hamiltonian system

$$
H(q, p)=T(q, p)+\Phi(q)
$$

where the position-dependent kinetic energy $T(q, p)$ is a quadratic form of the momenta and $\Phi(q)$ is a potential energy. The aim of the isokinetic thermostat is to constrain $T$ to a constant value $T_{0}^{2}>0$.

We define the isokinetic Hamiltonian $\mathcal{K}$ by

$$
\mathcal{K}(q, \pi)=e^{\Phi} T(q, \pi)-e^{-\Phi} T_{0}^{2}
$$

Via the non-canonical transformation,

$$
\pi=e^{-\Phi} p
$$

the level set $\mathcal{K}=0$ corresponds to constant kinetic energy $T=T_{0}^{2}$ in the system (1). Note that since $T$ is a quadratic form of the momenta, from $(3)$ we have

$$
T(q, \pi)=T\left(q, e^{-\Phi} p\right)=e^{-2 \Phi} T(q, p),
$$

and hence

$$
e^{\Phi} T(q, \pi)-e^{-\Phi} T_{0}^{2}=e^{-\Phi}\left(T(q, p)-T_{0}^{2}\right)
$$

Due to the Hamiltonian structure of the system, $\dot{\mathcal{K}}=0$ along the solutions of Hamilton's equations

$$
\begin{aligned}
& \dot{q}=\frac{\partial \mathcal{K}}{\partial \pi}=e^{\Phi} \frac{\partial T(q, \pi)}{\partial \pi} \\
& \dot{\pi}=-\frac{\partial \mathcal{K}}{\partial q}=-e^{\Phi} \frac{\partial \Phi}{\partial q} T(q, \pi)-e^{\Phi} \frac{\partial T(q, \pi)}{\partial q}-e^{-\Phi} \frac{\partial \Phi}{\partial q} T_{0}^{2}
\end{aligned}
$$




\section{B. Time scaling}

In this section, we show that the dynamics defined by equations (6) produce equivalent dynamics regardless of the value of $T_{0}^{2}$. We prove that a suitable scaling of time and momenta transforms (6) into equations with $T_{0}=1$.

Consider the scaling of time and momenta by

$$
s=T_{0} t, \quad \Pi=\frac{\pi}{T_{0}} .
$$

From (7) and

$$
d s=T_{0} d t
$$

it follows that

$$
\begin{aligned}
\frac{d q}{d s} & =\frac{d t}{d s} \frac{d q}{d t}=\frac{1}{T_{0}} \dot{q}, \\
\frac{d \Pi}{d s} & =\frac{d t}{d s} \frac{d \Pi}{d \pi} \frac{d \pi}{d t}=\frac{1}{T_{0}^{2}} \dot{\pi} .
\end{aligned}
$$

Using (6), (9) and

$$
T(q, \Pi)=\frac{1}{T_{0}^{2}} T(q, \pi)
$$

we can write the equations of motion in $s$ and $\Pi$ as

$$
\begin{aligned}
\frac{d q}{d s} & =\frac{1}{T_{0}} e^{\Phi} \frac{\partial T(q, \pi)}{\partial \pi}=T_{0} e^{\Phi} \frac{\partial T(q, \Pi)}{\partial \pi}=e^{\Phi} \frac{\partial T(q, \Pi)}{\partial \Pi} \\
\frac{d \Pi}{d s} & =-e^{\Phi} \frac{\partial \Phi}{\partial q} \frac{1}{T_{0}^{2}} T(q, \pi)-e^{\Phi} \frac{1}{T_{0}^{2}} \frac{\partial T(q, \pi)}{\partial q}-e^{-\Phi} \frac{\partial \Phi}{\partial q} \frac{1}{T_{0}^{2}} T_{0}^{2} \\
& =-e^{\Phi} \frac{\partial \Phi}{\partial q} T(q, \Pi)-e^{\Phi} \frac{\partial T(q, \Pi)}{\partial q}-e^{-\Phi} \frac{\partial \Phi}{\partial q}
\end{aligned}
$$

These equations correspond to the isokinetic Hamiltonian with $T_{0}=1$

$$
\mathcal{K}_{1}(q, \Pi)=e^{\Phi} T(q, \Pi)-e^{-\Phi},
$$

which is related to $\mathcal{K}$ via

$$
\mathcal{K}_{1}(q, \Pi)=\frac{1}{T_{0}^{2}} \mathcal{K}(q, \pi) .
$$

Clearly the dynamics of $\mathcal{K}$ and $\mathcal{K}_{1}$ is equivalent and only differs by scaling by a constant factor. 


\section{CHESNAVICH'S MODEL AND ROAMING}

In this section we introduce Chesnavich's model for the ion-molecule reaction $\mathrm{CH}_{4}^{+} \rightleftharpoons$ $\mathrm{CH}_{3}^{+}+\mathrm{H}$ and recall known results about roaming in this system. The model was introduced by Chesnavich ${ }^{6}$ to investigate the transition from vibration/librational motion of the H-atom in a deep potential well representing $\mathrm{CH}_{4}^{+}$to nearly-free rotation in a flat and rotationally symmetric region representing the dissociated $\mathrm{CH}_{3}^{+}+\mathrm{H}$.

\section{A. Chesnavich's Model Hamiltonian}

Chesnavich's $\mathrm{CH}_{4}^{+}$model is a Hamiltonian system with 2 degrees of freedom, consisting of a rigid $\mathrm{CH}_{3}^{+}$molecule and a mobile $\mathrm{H}$ atom. The system is defined by the Hamiltonian

$$
H\left(r, \theta, p_{r}, p_{\theta}\right)=\frac{1}{2} \frac{p_{r}^{2}}{\mu}+\frac{1}{2} p_{\theta}^{2}\left(\frac{1}{\mu r^{2}}+\frac{1}{I_{C H_{3}}}\right)+U(r, \theta),
$$

where $(r, \theta, \phi)$ are polar coordinates describing the position of the H-atom in a body-fixed frame attached to the $\mathrm{CH}_{3}^{+}$core (coordinate $\phi$ is ignorable in this model). The reduced mass

of the system $\mu=\frac{m_{\mathrm{CH}_{3}} m_{H}}{m_{C H_{3}}+m_{H}}$, where $m_{H}=1.007825 \mathrm{u}$ and $m_{C H_{3}}=3 m_{H}+12.0 \mathrm{u}$, and the moment of inertia of the rigid body $\mathrm{CH}_{3}^{+} I_{\mathrm{CH}_{3}}=2.373409 \mathrm{u} \AA^{2}$.

The potential $U(r, \theta)$ consists of a radial long range potential $U_{C H}$ and a short range potential $U_{\text {coup }}$ that models the short range anisotropy of the rigid $\mathrm{CH}_{3}^{+}$body.

$$
U(r, \theta)=U_{C H}(r)+U_{\text {coup }}(r, \theta) .
$$

It is characterised by two deep wells corresponding to bound $\mathrm{CH}_{4}^{+}$, two areas of high potential and a flat area beyond them as shown in Fig. 1.

The long range potential is

$$
U_{C H}(r)=\frac{D_{e}}{c_{1}-6}\left(2\left(3-c_{2}\right) e^{c_{1}(1-x)}-\left(4 c_{2}-c_{1} c_{2}+c_{1}\right) x^{-6}-\left(c_{1}-6\right) c_{2} x^{-4}\right),
$$

with parameter values as used in the original work $: x=\frac{r}{r_{e}}$, dissociation energy $D_{e}=47$ $\mathrm{kcal} / \mathrm{mol}$, equilibrium bond length $r_{e}=1.1 \AA, c_{1}=7.37$ and $c_{2}=1.61$. The short range hindered rotor potential $U_{\text {coup }}$ is defined by

$$
U_{\text {coup }}(r, \theta)=\frac{U_{e} e^{-a\left(r-r_{e}\right)^{2}}}{2}(1-\cos 2 \theta)
$$




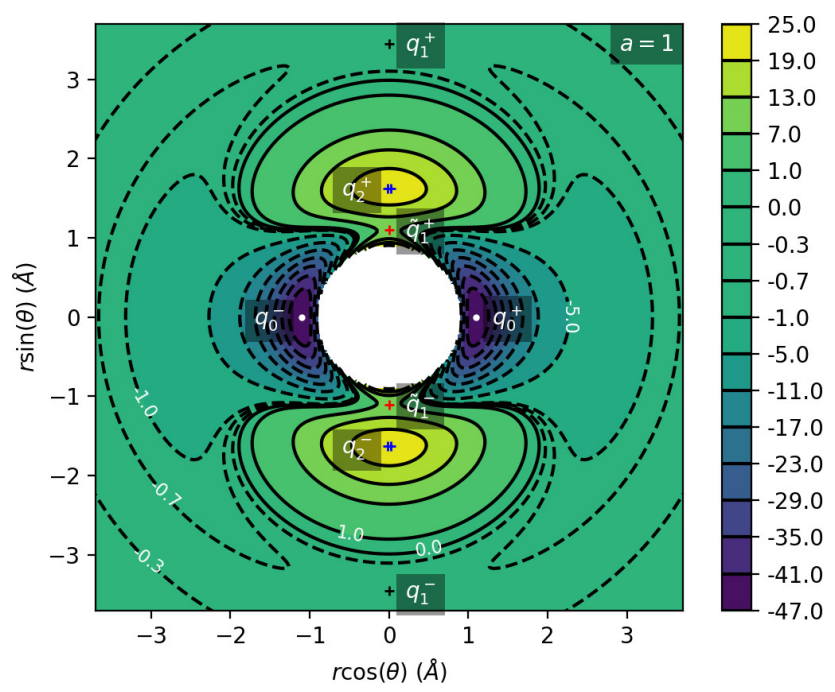

FIG. 1: Contour plot of Chesnavich's potential energy surface $U$ for $a=1$. Dashed lines correspond to $U<0$, solid lines correspond to $U \geq 0$. Contours correspond to values of potential shown on the colorbar right, with some values indicated in the plot.

where $U_{e}=55 \mathrm{kcal} / \mathrm{mol}$ is the equilibrium barrier height. The distance at which transition occurs from rotation to vibration is determined by the parameter $a$ (in $\AA^{-2}$ ). Various values of $a$ have been considered in previous works, namely $a=1^{6[12[16 \mid 17}, a=4^{6[17}$ and a range of values $0.7 \leq a \leq 8 \underline{13}$

The $\mathrm{CH}_{3}^{+}$is a symmetric top in Chesnavich's model. Although the range of coordinate $\theta$ is $0 \leq \theta \leq \pi$, in the planar (zero overall angular momentum) version of the model the range of $\theta$ is extended to $0 \leq \theta \leq 2 \pi$, and the potential has four-fold symmetry

$$
U(r, \theta)=U(r,-\theta)=U(r, \pi-\theta)=U(r, \pi+\theta)
$$

The potential admits four pairs of equilibrium points pairwise related by symmetry (18), as listed in Tab. I and shown in Fig. 1.

\section{B. Roaming in Chesnavich's Model}

As mentioned in Section If we are interested in the roaming mechanism wherein the hydrogen atom separates from the $\mathrm{CH}_{3}^{+}$core only to return to the vicinity of the core before dissociating. Here we review the dynamical definition of roaming as introduced in $\frac{17}{}$, which is based on periodic orbits as invariant structures having dynamical relevance. 


\begin{tabular}{c|c|c|c|c} 
Energy $\left(\mathrm{kcal} \mathrm{mol}^{-1}\right)$ & $r(\AA)$ & $\theta$ (radians) & Significance & Label \\
\hline-47 & 1.1 & 0 & potential well & $q_{0}^{+}$ \\
-0.63 & 3.45 & $\pi / 2$ & isomerisation saddle & $q_{1}^{+}$ \\
8 & 1.1 & $\pi / 2$ & isomerisation saddle & $\widetilde{q}_{1}^{+}$ \\
22.27 & 1.63 & $\pi / 2$ & local maximum & $q_{2}^{+}$
\end{tabular}

TABLE I: Equilibrium points of the potential $U(r, \theta)$.

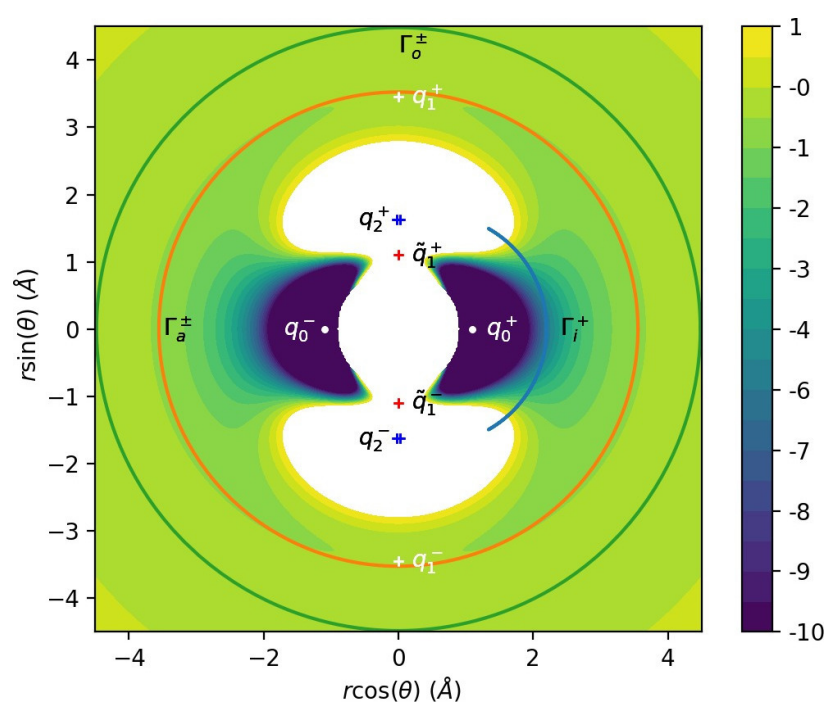

FIG. 2: Configuration space projections of inner $\left(\Gamma^{i}\right)$, middle $\left(\Gamma^{a}\right)$ and outer $\left(\Gamma^{o}\right)$ periodic orbits for $E=5$.

In the relevant energy interval $0 \leq E \leq 5$, there are three important families of periodic orbits $\frac{16}{}$. At any given fixed energy $0 \leq E \leq 5$, there are three pairs of important periodic orbits present in this system, pairwise related by symmetry (18), as shown in Fig. 2 , Therefore we refer to a continuum of periodic orbits parametrised by energy as a family of periodic orbits. We will refer to these families as the inner $\left(\Gamma^{i}\right)$, middle $\left(\Gamma^{a}\right)$ and outer $\left(\Gamma^{o}\right)$ periodic orbits. It is important to note that none of the orbits is directly related to a saddle point of the system.

Their significance is as follows:

- $\Gamma^{i}$ : Delimits the potential wells that correspond to $\mathrm{CH}_{4}^{+}$isomers. The orbits oscillate around the axes $\theta=0$ and $\theta=\pi$.

- $\Gamma^{a}$ : Two rotational orbits with opposite orientation - one clockwise, one counter- 
clockwise that are crucial for defining roaming.

- $\Gamma^{o}$ : Centrifugal barrier delimiting the region of dissociated states. Two rotational orbits with opposite orientation - one clockwise, one counter-clockwise.

Roaming was shown ${ }^{12}$ to disappear for $E \geq 2.5$. In the energy interval $0<E<2.5$, all of the above-mentioned periodic orbits are unstable. Define the dividing surfaces using $\operatorname{DS}^{i}$, $\mathrm{DS}^{a}$ and $\mathrm{DS}^{o}$ as the set of all phase space points $\left(r, \theta, p_{r}, p_{\theta}\right)$ that satisfy

$$
H\left(r, \theta, p_{r}, p_{\theta}\right)=E,
$$

and their configuration space projections $(r, \theta)$ coincide with the configuration space projections of $\Gamma^{i}, \Gamma^{a}$ and $\Gamma^{o}$ respectively. Due to the instability of the orbits, the resulting dividing surfaces do not admit local recrossing.

A roaming trajectory is then defined as a trajectory that crosses $\operatorname{DS}^{a}$ an odd number of times between leaving the potential well and dissociating. Trajectories that return to their region of origin cross $\mathrm{DS}^{a}$ an even number of times - isomerising trajectories returning to either of the potential wells, nonreactive trajectories return to dissociated states.

As explained in reference [12, the oscillatory nature of $\Gamma^{i}$ implies that $\mathrm{DS}^{i}$ consists of two spheres, while the rotational nature of $\Gamma^{a}$ and $\Gamma^{o}$ means that $\mathrm{DS}^{a}$ and $\mathrm{DS}^{o}$ are tori 18 . Each sphere can be divided using the corresponding periodic orbit into two hemispheres and each torus can be divided using both corresponding periodic orbits into two annuli 18 . All hemispheres and annuli are surfaces of unidirectional flux, for example all trajectories leaving the potential well cross the same (outward) hemisphere of $\mathrm{DS}^{i}$, while all trajctories entering the potential well cross the other (inward) hemisphere of $\mathrm{DS}^{i}$.

Roaming can hereby be reformulated as a transport problem in phase space. Every trajectory leaving the well must cross the outward hemisphere of $\mathrm{DS}^{i}$ and every trajectory that dissociates must cross the outward annulus of $\mathrm{DS}^{o}$. Dissociation of a $\mathrm{CH}_{4}^{+}$molecule is therefore equivalent to the transport of trajectories from the outward hemisphere of $\mathrm{DS}^{i}$ to the outward annulus of $\mathrm{DS}^{o}$. Roaming involves crossing the inward annulus of $\mathrm{DS}^{a}$, because between two crossings of the outward annulus trajectories must cross the inward annulus and vice versa.

Transport of trajectories in the neighbourhood of an unstable periodic orbit (or NHIM in general) is governed by invariant manifolds of this orbit 26 . It was shown $\frac{1213}{26}$ that the 


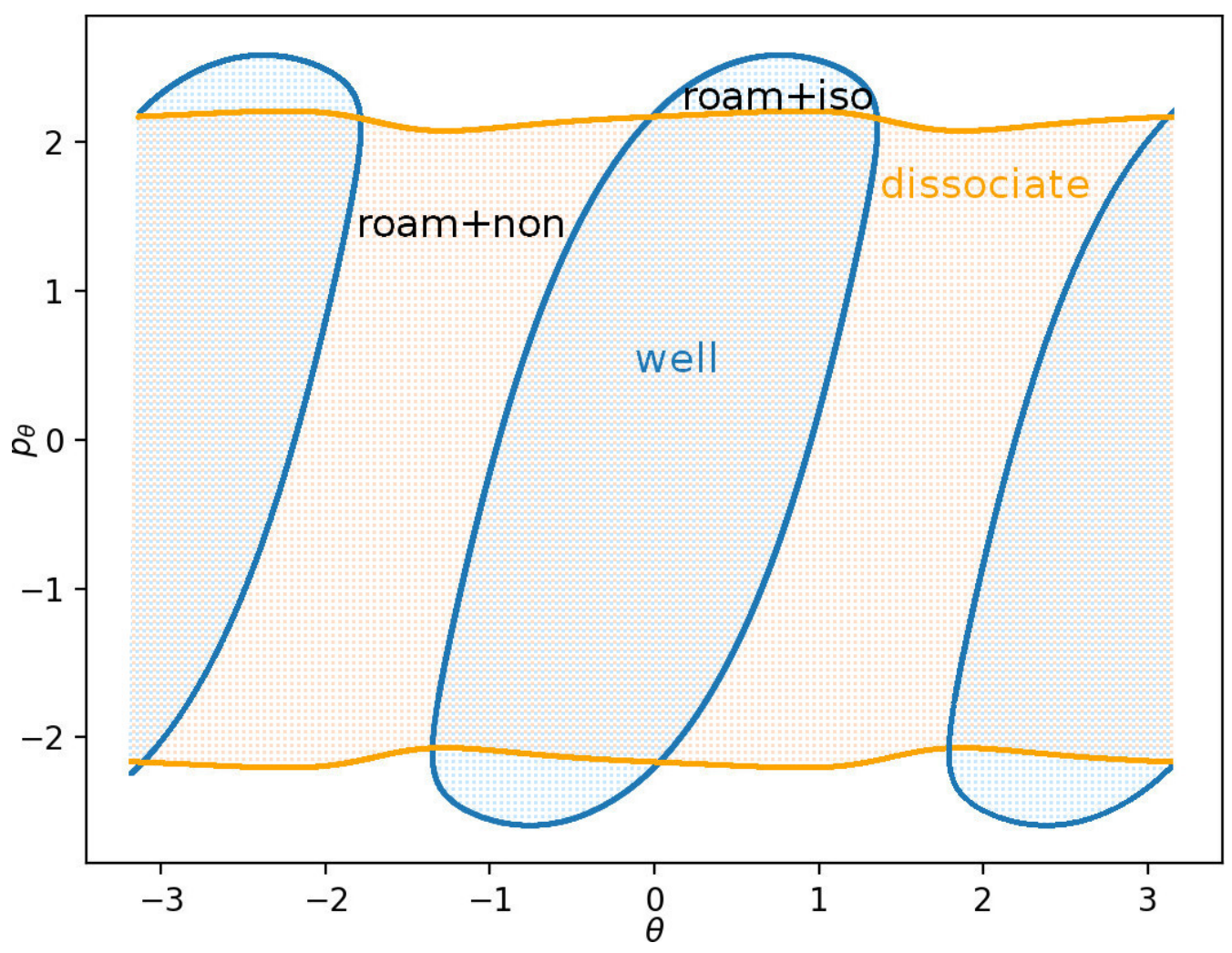

FIG. 3: Intersection of invariant manifolds of $\Gamma^{i}$ (blue) and $\Gamma^{o}$ (orange) with the outward annulus of $\mathrm{DS}^{a}$ for $E=1$. Trajectories that just left the potential well are shown in blue, immediately dissociating trajectories in orange. Roaming and isomerising trajectories in the blue area just left the well and do not dissociate immediately, while roaming and dissociating trajectories in the orange are dissociate immediately but did not just leave the potential wells.

roaming phenomenon involves a heteroclinic intersection of the invariant manifolds of $\Gamma^{i}$ and $\Gamma^{o}$. The condition $H\left(r, \theta=0, p_{r}>0, p_{\theta}=0\right)$ defines an invariant subsystem that consists of precisely one dissociating trajectory for every fixed $E>0$. Therefore if the invariant manifolds of $\Gamma^{i}$ and $\Gamma^{o}$ do not intersect, the former are contained in interior of the latter and each trajectory leaving the potential well dissociates immediately. An intersection assures that some trajectories leaving the well do not dissociate immediately and return to $\operatorname{DS}^{a}$ as illustrated in Fig. 3. This allows for roaming and isomerisation. 


\section{ISOKINETIC CHESNAVICH MODEL}

\section{A. Hamiltonian and periodic orbits for thermostatted Chesnavich model}

The isokinetic Hamiltonian $\mathcal{K}$ for Chesnavich's model is defined as follows:

$$
\mathcal{K}\left(r, \pi_{r}, \theta, \pi_{\theta}\right)=\frac{1}{2} e^{U}\left(\frac{\pi_{r}^{2}}{\mu}+\pi_{\theta}^{2}\left(\frac{1}{\mu r^{2}}+\frac{1}{I_{C H_{3}}}\right)\right)-\frac{1}{2} e^{-U}
$$

where $U=U(r, \theta)$ is Chesnavich's potential energy and

$$
\pi_{r}=e^{-U} p_{r}, \quad \pi_{\theta}=e^{-U} p_{\theta}
$$

The level set $\mathcal{K}=0$ corresponds to the surface of constant kinetic energy

$$
\frac{1}{2} \frac{p_{r}^{2}}{\mu}+\frac{1}{2} p_{\theta}^{2}\left(\frac{1}{\mu r^{2}}+\frac{1}{I_{C H_{3}}}\right)=\frac{1}{2},
$$

in system (14). Equations of motion in the isokinetic system are

$$
\begin{aligned}
\dot{r} & =\frac{\partial \mathcal{K}}{\partial \pi_{r}}=e^{U} \frac{\pi_{r}}{\mu} \\
\dot{\pi}_{r} & =-\frac{\partial \mathcal{K}}{\partial r}=-\frac{1}{2} e^{U} \frac{\partial U}{\partial r}\left(\frac{\pi_{r}^{2}}{\mu}+\pi_{\theta}^{2}\left(\frac{1}{\mu r^{2}}+\frac{1}{I_{C H_{3}}}\right)\right)+\frac{1}{2} e^{U} \frac{2}{\mu r^{3}} \pi_{\theta}^{2}-\frac{1}{2} e^{-U} \frac{\partial U}{\partial r} \\
\dot{\theta} & =\frac{\partial \mathcal{K}}{\partial \pi_{\theta}}=e^{U} \pi_{\theta}\left(\frac{1}{\mu r^{2}}+\frac{1}{I_{C H_{3}}}\right) \\
\dot{\pi}_{\theta} & =-\frac{\partial \mathcal{K}}{\partial \theta}=-\frac{1}{2} e^{U} \frac{\partial U}{\partial \theta}\left(\frac{\pi_{r}^{2}}{\mu}+\pi_{\theta}^{2}\left(\frac{1}{\mu r^{2}}+\frac{1}{I_{C H_{3}}}\right)\right)-\frac{1}{2} e^{-U} \frac{\partial U}{\partial \theta}
\end{aligned}
$$

To achieve greater numerical precision, it is preferable to integrate the equations of motion in $\left(r, p_{r}, \theta, p_{\theta}\right)$ coordinates instead of $\left(r, \pi_{r}, \theta, \pi_{\theta}\right)$. Equations (22) transform using (20) to

$$
\begin{aligned}
\dot{r} & =\frac{p_{r}}{\mu} \\
\dot{p}_{r} & =p_{r}\left(\frac{\partial U}{\partial r} \dot{r}+\frac{\partial U}{\partial \theta} \dot{\theta}\right)+\frac{1}{\mu r^{3}} p_{\theta}^{2}-\frac{\partial U}{\partial r} \\
\dot{\theta} & =p_{\theta}\left(\frac{1}{\mu r^{2}}+\frac{1}{I_{C H_{3}}}\right) \\
\dot{p}_{\theta} & =p_{\theta}\left(\frac{\partial U}{\partial r} \dot{r}+\frac{\partial U}{\partial \theta} \dot{\theta}\right)-\frac{\partial U}{\partial \theta}
\end{aligned}
$$

where we used the isokinetic constraint 21) equivalent to $\mathcal{K}=0$.

The potential $-\frac{1}{2} e^{-U}$ has the same critical points and characteristics as $U$, but the wells are considerably deeper and have steeper walls. In contrast to the microcanonical case, 


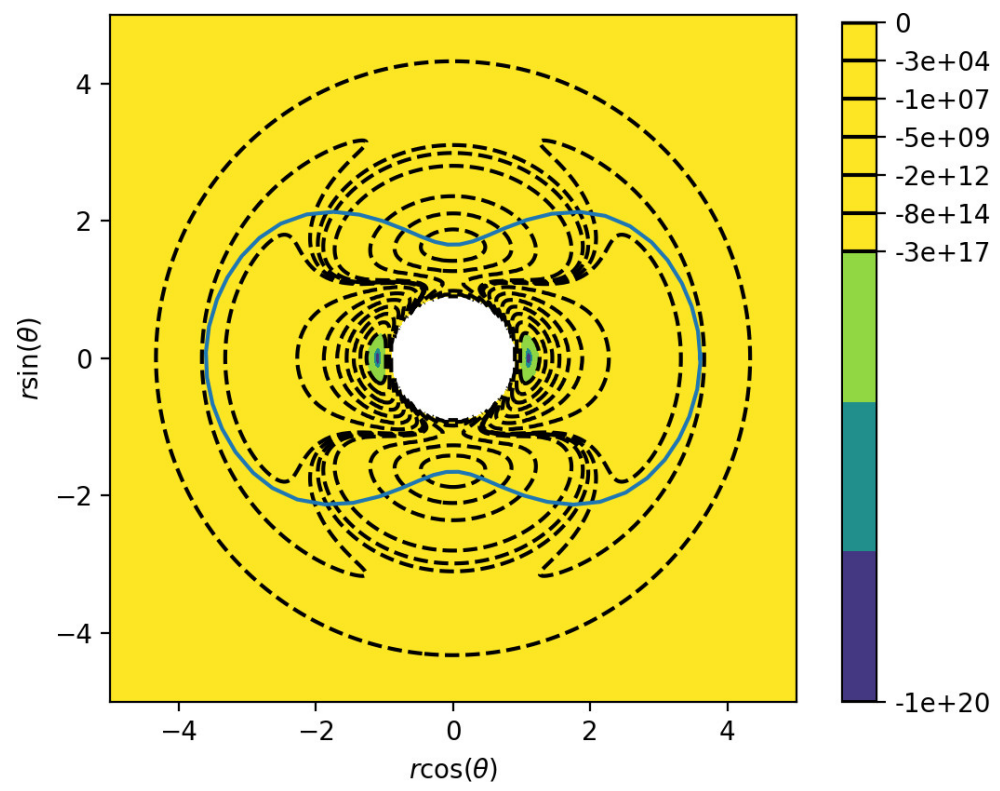

FIG. 4: Inner periodic orbit on the potential energy surface $-\frac{1}{2} e^{-U}$.

the isokinetic model only possesses two periodic orbits with period $2 \pi$ and due to constant nonzero kinetic energy does not admit self-retracing orbits (also referred to as brake orbits) such as $\Gamma^{i}$ introduced in Section III B. One of the periodic orbits delimits the potential wells, see Fig. 4, we therefore refer to it as the inner orbit.

The outer orbit, beyond which trajectories do not return and corresponds to the dissociated state of the molecule, is associated with a centrifugal barrier. It is rotationally symmetric and has a radius $r \approx 13.43$. Its existence can be proven using a similar argument as in the original system 12 : suppose $r$ is sufficiently large so that $U$ is effectively independent of $\theta$. Denote $r_{p o}$ the solution of

$$
\frac{1}{\mu r_{p o}^{3}} p_{\theta}^{2}-\frac{\partial U}{\partial r}=0
$$

Then the equations 23 admit a rotationally symmetric periodic orbit with $\dot{\theta}=$ const, provided

$$
\begin{gathered}
\dot{r}=0, \\
\dot{p}_{r}=0 .
\end{gathered}
$$

This is satisfied by the initial condition $r=r_{p o}, p_{r}=0$ and $p_{\theta}$ given implicitly by $\mathcal{K}=0$ for any $\theta$. The existence of $r_{p o}$ is guaranteed for the potential $U$ and any other potential with leading order term $-c r^{-(2+\varepsilon)}$ for large $r$, with $c>0$ and $\varepsilon>0$. 

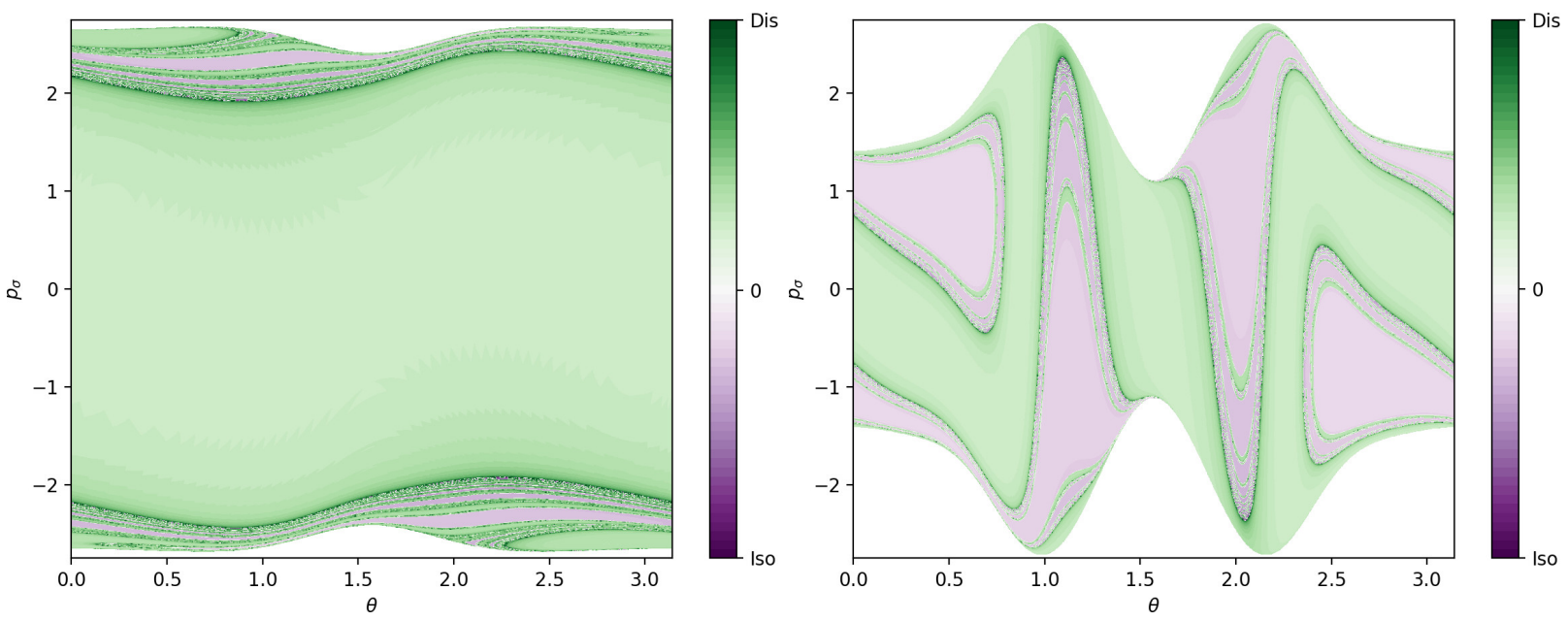

FIG. 5: Escape times for initial conditions on the outward annulus of $\mathrm{DS}^{a}$ in Chesnavich's model (left) and on the outward annulus of the inner DS in the isokinetic model (right). Short isomerisation times are shown in light purple, long isomerisation times in dark purple, short dissociation times in light green and long dissociation times in dark green.

Both these orbits are unstable, with the largest eigenvalue of the inner orbit under the return map being of the order $10^{21}$. This large instability poses a serious challenge to calculation of its invariant manifolds that guide trajectories in phase space.

\section{B. Classification of trajectories and roaming in the isokinetic Chesnavich model}

In this section we investigate dynamics in phase space and show the presence of roaming in the thermostatted Chesnavich model. Due to the strength of instability of the inner periodic

orbit, we visualise phase space structures using escape times and Lagrangian descriptors ${ }^{11115}$ on surfaces of section rather than calculating invariant manifolds themselves.

The two most natural surfaces of section for this system are: (i) $\theta=0, \dot{\theta}>0$ and (ii) the outward annulus of the inner dividing surface (DS). The latter surface of section is constructed as follows: let the configuration space projection of the inner periodic orbit be parametrised by

$$
r=\bar{r}(\theta) .
$$

Then all points in phase space satisfying (26) form a surface with coordinates $\theta$ and the 
canonically conjugate momentum ${ }^{1}$

$$
p_{\sigma}=p_{\theta}+\bar{r}^{\prime}(\theta) p_{r}
$$

We remark that this surface is, similarly to $\mathrm{DS}^{a}$ and $\mathrm{DS}^{o}$ in Sec. IIIB, a torus. On this torus, the value of $p_{r}^{2}$ is given implicitly by $\theta, p_{\sigma}$ and (21) and the sign of $p_{r}$ is chosen so that

$$
\dot{r}>\bar{r}^{\prime}(\theta)
$$

hence the outward direction on the inner DS.

The escape time is defined as the time a trajectory takes to escape the interaction region and reach a dissociated state beyond the outer orbit or either of the wells. Escape time plots obtained for initial conditions on the inner DS in the isokinetic model and on $\mathrm{DS}^{a}$ in the Hamiltonian Chesnavich model are shown in Fig. 5. The dynamics on $\mathrm{DS}^{a}$ is explained in Sec. IIIB. We can see that while the surfaces share a toroidal geometry, the distributions of escape times of trajectories on them are quite dissimilar. Most of the trajectories in the microcanonical case escape in the same manner as the prototypical dissociation trajectory $\theta=p_{\sigma}=0$ and more complicated escape dynamics are located near the edges of the surface of section. On the other hand, escape times for the isokinetic model reflect the effects of the constant kinetic energy constraint - note the nearly uniform dissociation around the local maximum at $\theta=\pi / 2$ and nearly uniform isomerisation regions around $\theta=1$ and $\theta=2$.

The boundary between dissociating and isomerising trajectories, as well as between direct dissociation and more complicated dynamics, consists of invariant manifolds. Fig. 66 shows escape times on the surface of section $\theta=0, \dot{\theta}>0$. Note the singularity in the plot separating a homogeneous area of relatively fast dissociation for large values of $p_{r}$ from more complicated dynamics for lower values of $p_{r}$. The singularity can also be seen in the line plot showing escape times on $r=6, \theta=0, \dot{\theta}>0$.

As it is the case in the microcanonical system, directly dissociating trajectories are guided by the stable invariant manifold of the outer periodic orbit across the phase space bottleneck associated with the outer periodic orbit. Directly dissociating trajectories are located above the stable invariant manifold in Fig. 6. All trajectories below the manifold correspond to roaming, isomerizing or nonreactive trajectories. As argued in Sec. IIIB, if there are trajectories on the inner DS that do not dissociate, the invariant manifolds of the inner and outer orbits must intersect. Fig. 5 shows regions of isomerization, therefore the manifolds 

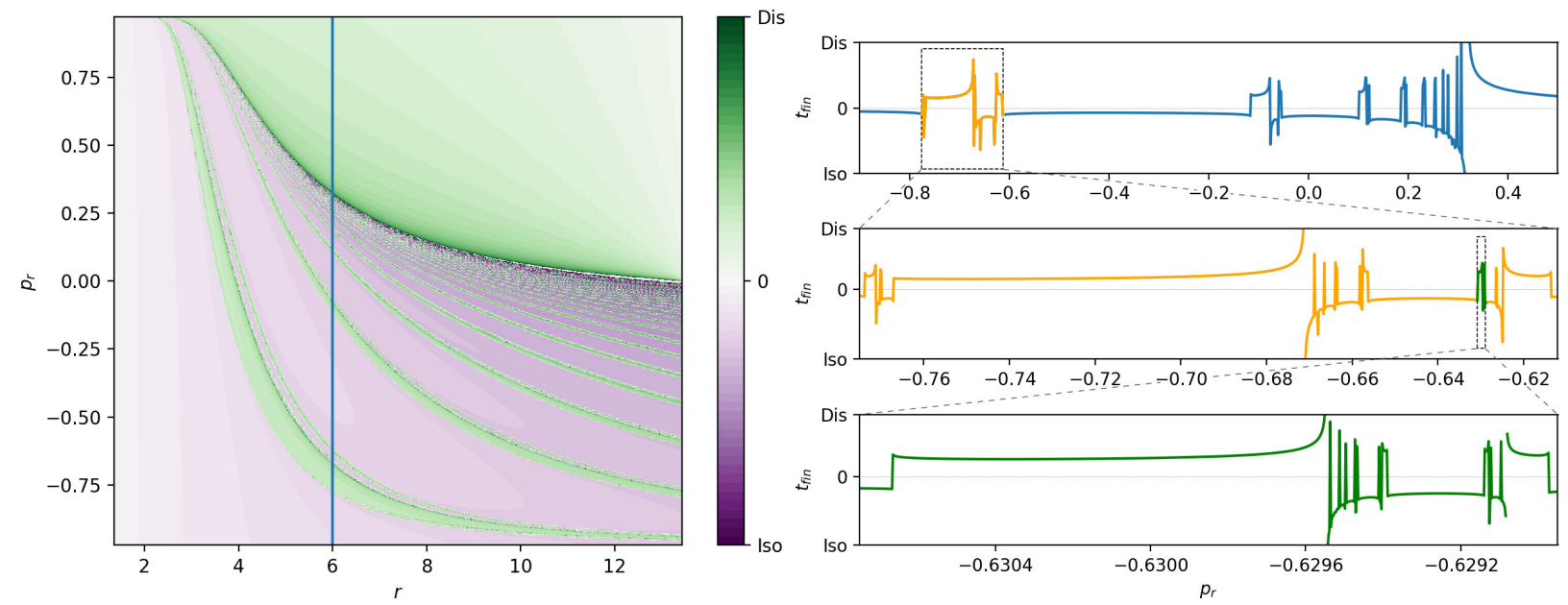

FIG. 6: Escape times for initial conditions on $\theta=0, \dot{\theta}>0$ and a the profile of the surface for $r=6$ with details of the fractal structure. Short isomerisation times are shown in light purple, long isomerisation times in dark purple, short dissociation times in light green and long dissociation times in dark green.

intersect and all dissociating trajectories in the fractal structures of complicated dynamics on the inner DS correspond to roaming trajectories.

The bands of isomerizing and dissociating trajectories form a fractal structure and can be classified by the number of rotations in $\theta$ before escaping from the interaction region. These too are separated by invariant manifolds on the inner and outer periodic orbits. The fractal structure from Fig. 6 can be understood using the configuration space projections of representative trajectories shown in Fig. 7. In general, trajectories exhibit two dynamical 'motifs': they can perform a rotation at large $r$ or interact with the areas of high potential near the local maxima at small $r$. Interaction with areas of high potential leads to escape out of the interaction region, capture in one of the potential wells, rotation in the same direction or rotation in the opposite direction. Trajectories are grouped by the sequence of these motifs in the fractal structure, for example trajectories that rotation by $\pi / 2$ and then dissociate or isomerise (see second panel in Fig. 7) are close to each other and well separated from trajectories that rotate by $\pi$. Each class could be denoted by a sequence of integers denoting the number of rotations (see third panel in Fig. 7) between interactions with the areas of high potential.

In this way we can find trajectories that perform any possible combination of rotations in the flat area of the interaction region and return any given number of times to the areas of 

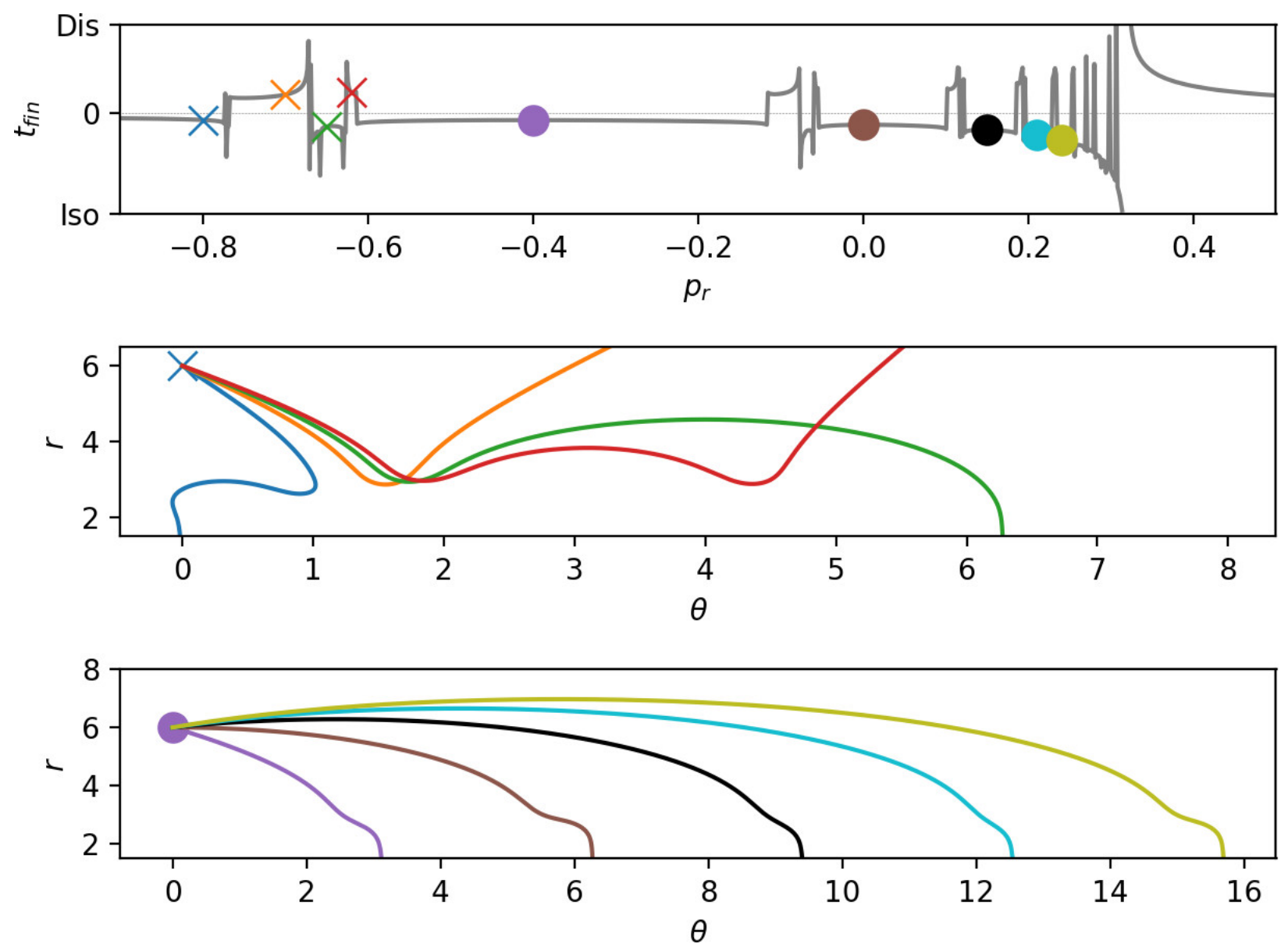

FIG. 7: Representative trajectories for different classes along $\theta=0, \dot{\theta}>0, r=6$.

high potential. In other words, regardless of the lack of self-retracing orbits in the interaction region, the scale of complicated dynamics exhibited by the isokinetic system is the same as in the microcanonical system.

It is important to note that the same results can be obtained using Lagrangian descriptors ${ }^{1115}$ as shown in Fig. 8. We present the plot for the forward Lagrangian descriptor

$$
\int_{0}^{40}\left(|\dot{r}|^{\frac{1}{2}}+\left|\dot{p_{r}}\right|^{\frac{1}{2}}+|\dot{\theta}|^{\frac{1}{2}}+\left|\dot{p_{\theta}}\right|^{\frac{1}{2}}\right) d t,
$$

with a cut-off at $r=1.1$ for numerical reasons. As opposed to escape times, this Lagrangian descriptor is well defined on invariant manifolds and attains a local minimum on them. Note that the invariant manifolds are visible for an integration time less than six times the period of the inner periodic orbit. The invariant manifolds are even more pronounced for the radial 

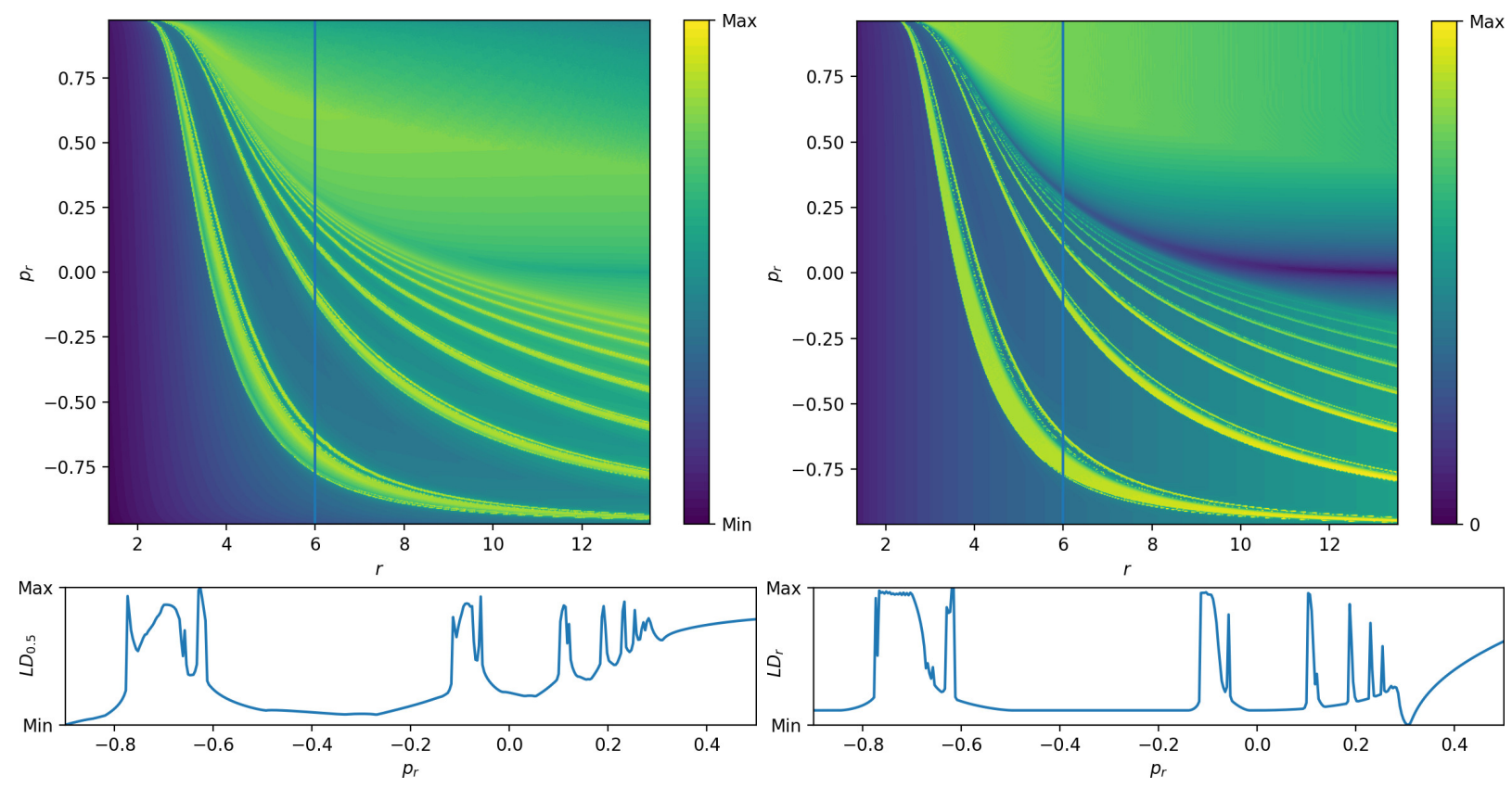

FIG. 8: Lagrangian descriptors (27) (left) and 28) (right) for initial conditions on $\theta=0, \dot{\theta}>0$ and their profiles for $r=6$.

gain Lagrangian descriptor

$$
\int_{0}^{40}|\dot{r}| d t .
$$

\section{CONCLUSIONS AND OUTLOOK}

In this paper we have studied the nonholonomic dynamics of a Hamiltonian system under the constraint of constant kinetic energy enforced by a Hamiltonian isokinetic thermostat. The thermostatted dynamics, if ergodic, generates a canonical (constant temperature) distribution in the system configuration space. Changing the characteristic temperature is equivalent to time scaling.

We further investigated the roaming mechanism in Chesnavich's model for an ionmolecule reaction subject to an isokinetic thermostat. Imposing the nonholonomic constraint does not prevent the system from exhibiting roaming dynamics, where the origin of the roaming mechanism turns out to be analogous to that found in the Hamiltonian case.

The nonexistence of so-called "brake orbits" in the isokinetic case (periodic orbits with points of zero velocity) leads to differences in the detailed phase space structure as compared 
to the microcanonical case, but the qualitative description of the roaming mechanism as a result of trapping in a region of phase space demarcated by invariant objects remains unchanged.

\section{FUNDING}

We acknowledge the support of EPSRC Grant no. EP/P021123/1 and Office of Naval Research (Grant No. N00014-01-1-0769).

\section{CONFLICT OF INTEREST}

The authors declare that they have no conflicts of interest.

* v.krajnak@bristol.ac.uk

$\dagger$ gse1@cornell.edu

$\ddagger$ s.wiggins@bristol.ac.uk

1 V. I. Arnold, Mathematical Methods of Classical Mechanics (Springer-Verlag, New York, 1978).

2 J. M. Bowman, Proc. Nat. Acad. Sci. 103, 16061 (2006).

3 J. M. Bowman and B. C. Shepler, Ann. Rev. Phys. Chem. 62, 531 (2011).

4 J. M. Bowman and A. G. Suits, Phys. Today 64, 33 (2011).

5 J. M. Bowman, Mol. Phys. 112, 2516 (2014).

6 W. J. Chesnavich, J. Chem. Phys. 84, 2615 (1986).

7 P. Collins, G. S. Ezra, and S. Wiggins, J. Chem. Phys. 133, 014105 (2010).

8 C. P. Dettmann and G. P. Morriss, Phys. Rev. E 54, 2495 (1996).

9 G. S. Ezra and S. Wiggins, J. Phys. A 42, 042001 (2008).

10 G. S. Ezra and S. Wiggins, Int. J. Bifurcation Chaos 29, 1950025 (2019).

11 J. A. Jimenez Madrid and A. M. Mancho, Chaos 19, 013111 (2009).

12 V. Krajňák and H. Waalkens, J. Math. Chem. (2018).

13 V. Krajňák and S. Wiggins, J. Chem. Phys. 149, 094109 (2018).

14 M. Litniewski, J. Phys. Chem. 97, 3842 (1993). 
15 C. Lopesino, F. Balibrea, S. Wiggins, and A. M. Mancho, Comm. Nonlinear Sci. Numer. Simulat. 27, 40 (2015).

16 F. A. Mauguière, P. Collins, G. S. Ezra, S. C. Farantos, and S. Wiggins, Chem. Phys. Lett. 592, 282 (2014).

17 F. A. L. Mauguière, P. Collins, G. S. Ezra, S. C. Farantos, and S. Wiggins, J. Chem. Phys. 140, $134112(2014)$

18 F. A. L. Mauguiere, P. Collins, Z. C. Kramer, B. K. Carpenter, G. S. Ezra, S. C. Farantos, and S. Wiggins, J. Chem. Phys. 144, 054107 (2016).

19 F. A. Mauguiere, P. Collins, Z. C. Kramer, B. K. Carpenter, G. S. Ezra, S. C. Farantos, and S. Wiggins, Ann. Rev. Phys. Chem. 68 (2017).

20 P. Minary, G. J. Martyna, and M. E. Tuckerman, J. Chem. Phys. 118, 2510 (2003).

21 P. Minary, G. J. Martyna, and M. E. Tuckerman, J. Chem. Phys. 118, 2527 (2003).

22 T. Morishita, J. Chem. Phys. 119, 7075 (2003).

23 G. P. Morriss and C. P. Dettmann, Chaos 8, 321 (1998).

24 A. G. Suits, Acc. Chem. Res. 41, 873 (2008).

25 D. Townsend, S. A. Lahankar, S. K. Lee, S. D. Chambreau, A. G. Suits, X. Zhang, J. Rheinecker, L. B. Harding, and J. M. Bowman, Science 306, 1158 (2004).

26 T. Uzer, C. Jaffé, J. Palacián, P. Yanguas, and S. Wiggins, Nonlinearity 15, 957 (2002).

27 H. Waalkens, R. Schubert, and S. Wiggins, Nonlinearity 21, R1 (2008).

28 S. Wiggins, L. Wiesenfeld, C. Jaffe, and T. Uzer, Phys. Rev. Lett. 86(24), 5478 (2001).

29 S. Wiggins, Reg. Chaot. Dyn. 21, 621 (2016).

30 R. D. van Zee, M. F. Foltz, and C. B. Moore, J. Chem. Phys. 99, 1664 (1993). 